\title{
Efecto de la estructura del cultivo de palma de aceite Elaeis guineensis (Arecaceae) sobre la diversidad de aves en un paisaje de la Orinoquía colombiana
}

\author{
Diana Patricia Tamaris-Turizo ${ }^{1,2 *}$, Hugo Fernando López-Arévalo ${ }^{1} \&$ Nabhi Romero Rodríguez ${ }^{1}$ \\ 1. Grupo en Conservación y Manejo de Vida Silvestre, Instituto de Ciencias Naturales, Universidad Nacional de \\ Colombia, Bogotá; dptamarist@unal.edu.co, hflopeza@unal.edu.co,nromeror@unal.edu.co \\ 2. Facultad de Ciencias Básicas, Universidad del Magdalena. \\ * Correspondencia
}

Recibido 06-III-2017. Corregido 20-VII-2017. Aceptado 22-VIII-2017.

\begin{abstract}
Effect of Oil Palm crop structure Elaeis guineensis (Arecaceae) on bird diversity in a tropical landscape of Colombian Orinoquia. The expansion of oil palm plantations has caused drastic changes in natural ecosystems and modifications in the vegetation structure, reducing available habitat for native bird species. We studied birds associated with oil palm plantations plots of different palm size classes, in the foothills of the Colombian Orinoquia region, from August 2013 and February 2014. The palm size clases were three: small up to $6 \mathrm{~m}$ height; medium up to $10 \mathrm{~m}$; and large up to $15 \mathrm{~m}$; in each plot we counted birds through line transects, and compared their bird composition, richness, and abundance, and described trophic group structure. We recorded a total of 468 individuals, belonging to 44 bird species and 23 families. Gymnomystax mexicanus, Crotophaga ani and Milvago chimachima were the most abundant species, the first tow representing $4.5 \%$ of the abundant species; $25 \%$ had intermediate abundances and $70.4 \%$ were rare in the plantations area. In terms of the abundance of bird species, medium and large palms plots were more similar. Bird community found in plots with large palms had the highest diversity and uniformity ( 2.63 and 0.8907 , respectively), while in those of small palms it was a greater dominance and abundance of individual birds ( 0.1825 and 252 , each one). For the entire plantation, insectivorous and carnivorous birds predominated, and had greater association with small and large palms. Oil palm stands serve as suitable habitats for a small number of widespread bird species, and the height does not seem to be an important factor, that may meaningfully benefit the composition, richness and abundance of bird species associated with the plantations. Rev. Biol. Trop. 65 (4): 1569-1581. Epub 2017 December 01.
\end{abstract}

Key words: bird community, agricultural practice, Orinoquia, structural complexity, oil palm, trophic groups.

La complejidad estructural es un factor que regula la riqueza y diversidad de aves terrestres (MacArthur \& MacArthur, 1962; Hilden, 1965; Cousin \& Phillips, 2008). La asociación y organización de las aves en un ecosistema, también dependen de los recursos disponibles para el forrajeo, la reproducción, el refugio frente a las adversidades del clima, la presencia de parásitos y depredadores (Hilden, 1965; Cousin $\&$ Phillips, 2008). Por lo tanto, las actividades humanas que conducen a los cambios en la composición y estructura de la vegetación, pueden afectar la persistencia de aves por la insuficiencia de recursos, aislamiento entre hábitats o niveles elevados de parasitismo y depredación (Miller \& Cale, 2000).

La Orinoquía colombiana se ha destacado como una de las regiones que alberga mayor riqueza de aves en Colombia, los registros incluyen 761 especies de aves que representan aproximadamente el $19 \%$ de las especies a nivel mundial y el $47.6 \%$ de las especies registradas para el país (McNish, 2007; Rangel-Ch \& Minorta-Cely, 2014). Sin embargo, esta diversidad de aves está en riesgo debido al extensivo y acelerado remplazo de la vegetación nativa transformada en un monocultivo de palma de aceite, que viene generando cambios 
en la composición y estructura de la vegetación (Umaña-Villaveces, Murillo, Restrepo-Calle \& Álvarez-Rebolledo, 2009). El sector palmero considera la Orinoquía como una región con gran potencial para la expansión del cultivo de palma de aceite (Andrade et al., 2009). Estas plantaciones se han establecido en la mencionada región desde los años 70's y aportan cerca del $30 \%$ de la producción nacional de aceite de palma (Fedepalma, 2007; Andrade et al., 2009). $\mathrm{Su}$ producción y expansión es una respuesta de la alta demanda de biodiesel, productos comestibles y otros productos no comestibles, lo cual resulta en acelerados cambios ambientales (Koh \& Ghazoul, 2008). Actualmente, el gobierno colombiano fomenta la ampliación de la frontera agrícola a través del programa Desarrollo Rural con Equidad (DRE) y del Incentivo a la Capitalización Rural (ICR) para pequeños y medianos productores.

Los cultivos de palma de aceite son sistemas uniformes, el establecimiento de una plantación implica la remoción de la vegetación nativa (Fitzherbert et al., 2008), las variaciones estructurales del cultivo se reducen a la vegetación epífita que crece sobre troncos, la vegetación que crece en el suelo y el sotobosque, cuando forma parte del manejo del cultivo (Nájera \& Simonetti, 2010; Luskin \& Potts, 2011). La siembra de palma de aceite a escala industrial, ha generado impactos negativos en diversos componentes de la biodiversidad (Aratrakorn, Thunhikorn \& Donald, 2006; Fitzherbert et al., 2008; Edwards et. al., 2010; García-Ulloa, Sloan, Pacheco, Ghazoul \& Pin Koh, 2012), a través de la degradación de hábitat, destrucción y reemplazamiento de la vegetación nativa, que en el caso de la Orinoquía afecta a bosques húmedos, bosques secos, bosques de galería, pastizales y humedales (Blydenstein, 1967; Bolwing, Pomeroy, Tushabe \& Mushabe, 2006; Pinzón, Pardi, Trejos \& Midori, 2009), lo que genera cambios en la composición y estructura de la vegetación (Correa \& Stevenson, 2010).

Estos cultivos son dominados por un reducido número de aves comunes que tienen amplio rango de distribución, mientras que todas aquellas especies asociadas a bosque quedan confinadas a los relictos de vegetación nativa (Aratrakorn et al., 2006; Bolwing et al., 2006; Achondo et al., 2011). Las aves asociadas a estos monocultivos habitan diferentes espacios de la plantación y de acuerdo a la edad de la palma se han encontrado especies distintas. Así, por ejemplo, se han encontrado especies de importancia de conservación (endémicas y amenazadas) en palmares jóvenes cercanos a bosques nativos (De Chenon \& Susanto, 2006). La riqueza de especies de aves puede aumentar cuando hay mayor complejidad estructural dentro del cultivo, p.e. epífitas, plantas presentes en el suelo, sotobosque y árboles nativos dentro de la plantación (Fitzherbert et al., 2008; Cagod \& Nuñeza, 2012), pero también por el efecto de borde, cuando hay disponibilidad de fragmentos de bosque, y por la complejidad estructural de la vegetación cercana al cultivo (Cagod \& Nuñeza, 2012).

El gremio palmicultor adelantan el proyecto Conservación de la Biodiversidad en las Zonas de Cultivo de Palma de Aceite como una iniciativa orientada a la sostenibilidad ambiental y que busca promover el principio de la responsabilidad con el medio ambiente y conservación de recursos naturales y la biodiversidad (RSPO, 2014), sin embargo, en Colombia hace falta rigor para que las prácticas de agronegocios se realicen de forma sostenible. Con el fin de aportar información a la discusión sobre el impacto ambiental de los agronegocios, se estudió la composición, riqueza y abundancia de aves, así como la estructura de los grupos tróficos, y se comparó con tres clases de altura del cultivo de palma de aceite, en la región oriental de Colombia.

\section{MATERIALES Y MÉTODOS}

Área de estudio: El estudio se realizó en dos fincas con cultivos de palma de aceite Tierra Viva (TIV) y La Mejorana (MEJ). TIV pertenece al municipio de San Martín de los Llanos, tiene un área de 1400 ha y está ubicada a los $03^{\circ} 37^{\prime} 51^{\prime}$ " $N$ y $73^{\circ} 20^{\prime} 59^{\prime}$ ' W, y a 420 m.s.n.m. MEJ se encuentra en el municipio 
de Acacias, la finca tiene un área de 1480 ha y se encuentra ubicada a los $03^{\circ} 57^{\prime} 37.18^{\prime \prime} \mathrm{N}$ y 7337'32.93" W, y a 498 msnm. Estos municipios pertenecen al departamento del Meta (Llanos Orientales de Colombia).

$\mathrm{El}$ área de estudio forma parte de la unidad fisiográfica piedemonte depositacional, derivado de rocas terciarias y cretáceas (Casanare y Meta), con un área total de 2532526 ha, que corresponde al $6.7 \%$ del área total de la cuenca del Orinoco, dentro del zonobioma húmedo tropical conformado por 67 ecosistemas que se localizan entre los 50 a 1100 m.s.n.m. (Correa, Ruiz \& Arévalo, 2005). Las formaciones vegetales más representativas son bosques de estrato arbóreo con elementos de 18 y $30 \mathrm{~m}$ de altura, herbazales con elementos leñosos, pastizales con resto de bosque, pastizales con elementos de $20 \mathrm{~cm}$ de altura donde domina Paspalum carinatum y palmares con predominio de Socratea exorrhiza e Iriartea deltoidea (Blydenstein, 1967; Rangel-Ch \& Minorta-Cely, 2014). Este tipo de vegetación se ha modificado desde el siglo XX por la actividad ganadera y por el establecimiento y consolidación de la agricultura comercial basada en cultivos de palma de aceite, arroz, maíz, sorgo, soya, frutales, entre otros (Rippstein, Escobar \& Motta, 2001; Mora Fernández, Castellanos, Cardona, Pinzón \& Vargas, 2011).

Censos de aves: Se hicieron observaciones de aves durante cuatro días consecutivos en junio, agosto y noviembre de 2013, y en febrero y julio de 2014, en 18 lotes (parcelas): seis de palmas pequeñas (de 2 a $6 \mathrm{~m}$ ), seis de palmas medianas (7-10 m) y seis de palmas grandes $(11-15 \mathrm{~m})$. Se definieron estas clases de altura teniendo en cuenta que la altura máxima promedio de una palma en una plantación es de 15 a $18 \mathrm{~m}$ y que a partir de este rango de altura puede haber reemplazo de las palmas, por la dificultad para la cosecha de frutos (ASD, 2006). Los meses de muestreo coincidieron con eventos de sequía (febrero) y lluvia (julio, agosto y noviembre), correspondientes al régimen unimodal biestacional característico de la región (Minorta-Cely \& Rangel-Ch, 2014). El área de los lotes fue variable (23.3 a 48.7 ha), incluyendo 160 palmas/ha. La altura de las palmas se consideró desde la base del tronco hasta el extremo superior del dosel (Luskin \& Potts, 2011). En general, los troncos tuvieron plantas epífitas, y en ambas localidades se implementaron técnicas manuales y mecánicas para el control de hierbas y prevenir el crecimiento de vegetación arbustiva.

Las aves se contaron con el método de transecto lineal de ancho variable (Buckland, Anderson, Burnham \& Laake, 1993). Para ello, en cada lote, se estableció una línea de $500 \mathrm{~m}$, y se censaron todas las aves dentro del lote, en cada caso se tuvo en cuenta la distancia del ave con respecto al transecto, lo cual se usó para calcular la densidad de individuos por $\mathrm{km}^{2}$ para todo el cultivo y por clase de altura. Los transectos se ubicaron teniendo en cuenta el tamaño del lote y la accesibilidad a éste, manteniendo una distancia mínima de $800 \mathrm{~m}$ con respecto al transecto más cercano, con el fin de garantizar la independencia de las observaciones. Este método de conteo es recomendable en hábitats de baja densidad con una estructura uniforme (Bibby, Jones \& Marsden, 2000). Cada transecto se recorrió seis veces durante el periodo de estudio, completando 108 recorridos en total, pero para el análisis de datos se tuvieron en cuenta los recorridos donde hubo registro de especies (81 recorridos). El orden para recorrer los transectos se escogió aleatoriamente en cada visita, para evitar sesgos en los conteos según la hora del día (Bibby et al., 2000), y se dedicó 40 minutos en cada recorrido. En el registro se incluyeron las especies observadas y escuchadas (Bibby et al., 2000). Un observador tomó los registros en la mañana (06:00 a 10:30) y en la tarde (15:00 a 17:30), y se invirtió un esfuerzo de muestreo mínimo diario de $2.5 \mathrm{~km} /$ día; se utilizó binoculares Nikon Action de alcance $8 \times 40 \mathrm{~mm}$. Durante las observaciones no se tuvieron en cuenta los individuos de paso o que sobrevolaron el sitio de estudio por encima de los 20 m (Codesido \& Bilenca, 2004). Todas las especies observadas fueron asignadas a una categoría de grupo trófico, la cual se basó tanto en las observaciones de campo como 
en la literatura disponible (Hilty \& Brown 2001; Restall, Rodner \& Lentino, 2006).

Se construyó una curva de acumulación de especies utilizando los registros de aves (global para el cultivo y discriminado por clases de altura) para identificar si el esfuerzo de muestreo fue lo suficientemente representativo. También se estimó el número de especies posibles con los estimadores de riqueza Chaol y ACE. Se graficaron los valores de abundancia de cada especie (n), registradas en cada clase de altura, para visualizar la proporción de las aves dentro de la plantación.

La diversidad de la comunidad de aves fue analizada a partir de índices de diversidad (Magurran, 1988; Moreno, 2001). La diversidad $\alpha$ fue el número total de especies obtenido en palmas de cada clase de altura, también se calculó el índice de diversidad de Brillouin $(\mathrm{H})$, el índice de equitatividad Pielou $(\mathrm{J})$ y el índice de dominancia de Simpson $(\lambda)$, mientras que la diversidad $\beta$ (recambio de especies entre palmas grandes: $G$, medianas: $M$ y pequeñas: P) se calculó, a partir de los valores de riqueza (S) y de abundancia (N) en cada clase de altura, con el Coeficiente de Similaridad, el índice de Whittaker $\left(\beta_{\mathrm{W}}\right)$ y el índice de Complementareidad según lo describe Magurran (1988). También se generó el modelo de distribución de abundancias (Magurran, 1988) para describir la estructura de la comunidad en términos de la abundancia proporcional de las especies.

A partir de un coeficiente de correlación (Krebs, 1999) se analizó el grado de similitud teniendo en cuenta la abundancia de las especies, entre cultivos de palmas grandes, medianas y pequeñas, utilizando el programa PAST 3. Un análisis de correspondencia rectificado (DCA) se usó para identificar si los grupos tróficos tuvieron una segregación con respecto a las clases de altura de la plantación, asimismo, se aplicó la prueba $\mathrm{Chi}^{2}$ para identificar posibles diferencias significativas de la abundancia de aves de cada grupo trófico, con respecto a las clases de altura de la palma. Finalmente, se utilizó la prueba no paramétrica Friedman para identificar si existían diferencias significativas de la abundancia de individuos entre las clases de altura.

\section{RESULTADOS}

Durante el estudio se registraron 468 individuos asociados al cultivo de palma, agrupados en 44 especies, 23 familias y 14 órdenes. Las especies más abundantes dentro del cultivo de palma fueron Gymnomystax mexicanus, Crotophaga ani y Milvago chimachima. Las dos primeras fueron las especies más dominantes en palma pequeña, mientras que $M$. chimachima fue la especie más dominante en lotes de palma mediana y grande (Fig. 1).

La curva de acumulación de especies total (Fig. 2A) no se estabilizó, lo cual indica que el censo que se hizo en campo no fue suficiente para proporcionar una caracterización completa de las aves presentes en el cultivo de palma de aceite, aunque la riqueza observada (44 especies) corresponde al $88 \%$ de la riqueza calculada por el estimador Chaoly ACE (48 y 50 especies, respectivamente). Observamos que el $70.4 \%(n=16)$ de las especies fueron raras (aquellas que estuvieron representadas por uno o dos individuos), lo que generó un efecto ascendente en las curvas de acumulación. Un comportamiento parecido al anterior se observó en la curva de acumulación de especies para cada clase de altura (Fig. 2B). La riqueza observada en cada clase de altura correspondió al $96(n=27), 80(n=20)$ y $93 \%(n=30)$ con respecto a la riqueza calculada por el estimador de riqueza Chao 1 en palmas grandes, medianas y pequeñas, respectivamente.

La similitud a partir del coeficiente de correlación entre pares de sitios indicó que las palmas grandes y medianas fueron más similares entre sí $(0.816)$ en términos de la abundancia proporcional de las especies (Cuadro 1). La densidad de individuos fue mayor en las palmas pequeñas (6.9 ind. $/ \mathrm{km}^{2}, \mathrm{SD} \pm$ 2.65), reduciéndose a medida que aumentó la altura (4.0 ind./ $\mathrm{km}^{2}, \mathrm{SD} \pm 8.5$ y 2.8 ind./ $\mathrm{km}^{2}, \mathrm{SD} \pm 6.6$, en palmas medianas y grandes, respectivamente). Los modelos de la distribución de abundancia de las especies fueron 


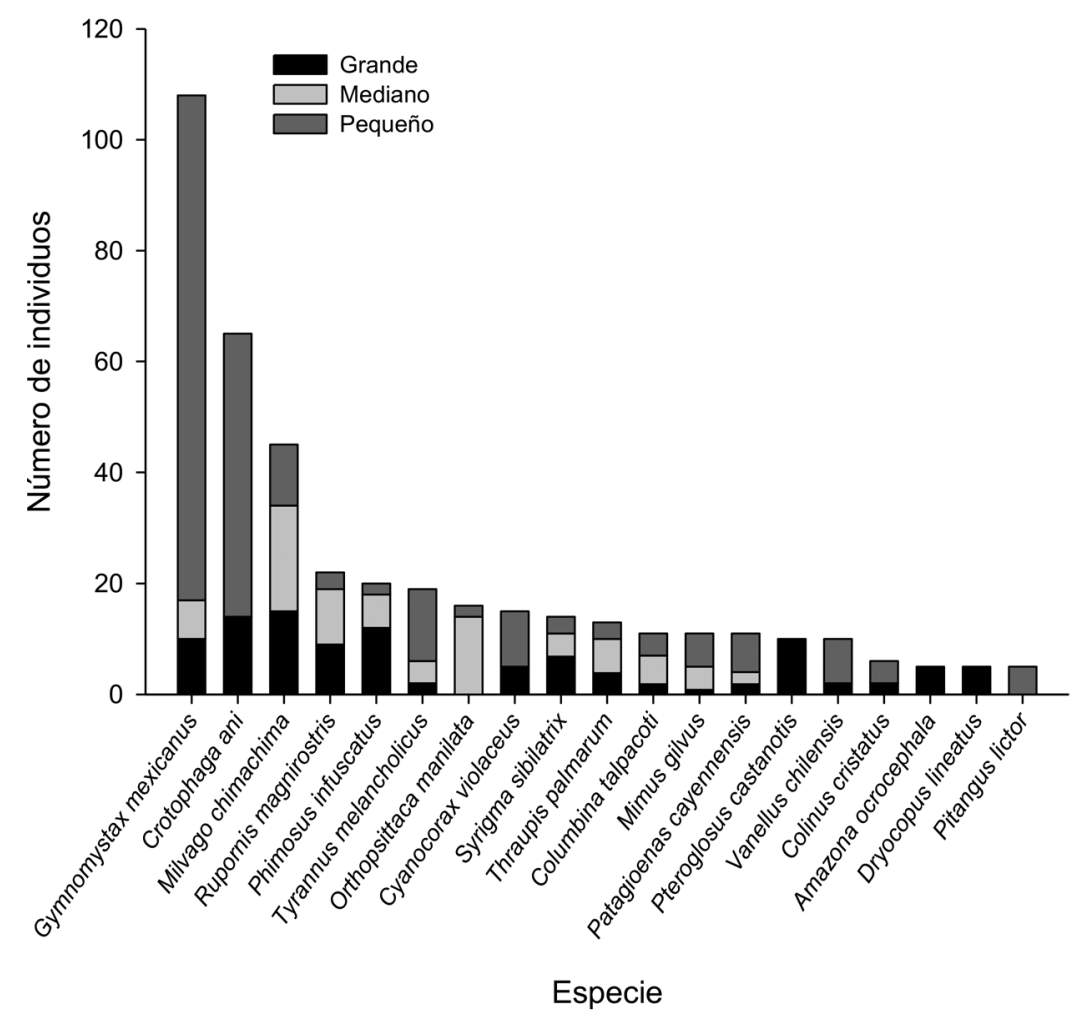

Fig. 1. Distribución de la abundancia de especies en cada clase de altura del cultivo de palma de aceite.

Fig. 1. Abundance distribution of species in each height class of Oil Palm crop.

\section{CUADRO 1}

Cálculos de diversidad de aves asociadas a cultivos de palmas grandes, medianas y pequeñas del cultivo de palma de aceite

TABLE 1

Diversity values of birds associated to large, medium and small palms of the Oil Palm crop

\begin{tabular}{lccccccccc} 
& $\begin{array}{c}\text { Riqueza } \\
(\mathrm{S})\end{array}$ & $\begin{array}{c}\text { Abundancia } \\
(\mathrm{N})\end{array}$ & $\begin{array}{c}\text { Diversidad } \\
\text { (Brillouin) }(\mathrm{H})\end{array}$ & $\begin{array}{c}\text { Uniformidad } \\
\text { Pielou }\left(\mathrm{J}^{\prime}\right)\end{array}$ & $\begin{array}{c}\text { Dominancia } \\
\text { Simpson }(\lambda)\end{array}$ & $\begin{array}{c}\text { Pares de } \\
\text { biotas * }\end{array}$ & $\begin{array}{c}\text { Coeficiente de } \\
\text { Similaridad }\end{array}$ & $\begin{array}{c}\text { Índice de } \\
\text { Whittaker }\left(\beta_{\mathrm{W}}\right)\end{array}$ & $\begin{array}{c}\text { Índice de Comple- } \\
\operatorname{mentareidad}\left(\mathrm{C}_{\mathrm{AB}}\right)\end{array}$ \\
Grande & 27 & 124 & 2.63 & 0.8907 & 0.06764 & $\mathrm{G}-\mathrm{M}$ & 0.816 & 1.95 & 0.53 \\
Mediano & 20 & 92 & 2.27 & 0.8567 & 0.1028 & G-P & 0.44 & 1.60 & 0.64 \\
Pequeño & 30 & 252 & 2.21 & 0.702 & 0.1825 & M-P & 0.357 & 1.83 & 0.61 \\
\hline
\end{tabular}

*G: grande, M: mediano, P: pequeño. / G: large, M: medium, P: small.

semejantes en las tres clases de altura del cultivo de palma y no hubo diferencia significativa entre clases de altura (Fig. 3, Friedman= $1,67, \mathrm{p}=0.3670$ ). Encontramos que el $70 \%$ de la comunidad fueron especies raras (especies con 10 o menos individuos), el $25 \%$ tuvieron abundancias intermedias (especies con 11 a 49 individuos) y el $4.5 \%$ de las especies fueron abundantes dentro de la plantación (especies con 50 o más individuos), lo cual se ajusta al modelo Serie Logarítmica. Los índices de diversidad demostraron que la palma grande tuvo mayor equidad $(\mathrm{H})$ y uniformidad $\left(\mathrm{J}^{\prime}\right)$ en la abundancia proporcional de las especies, 

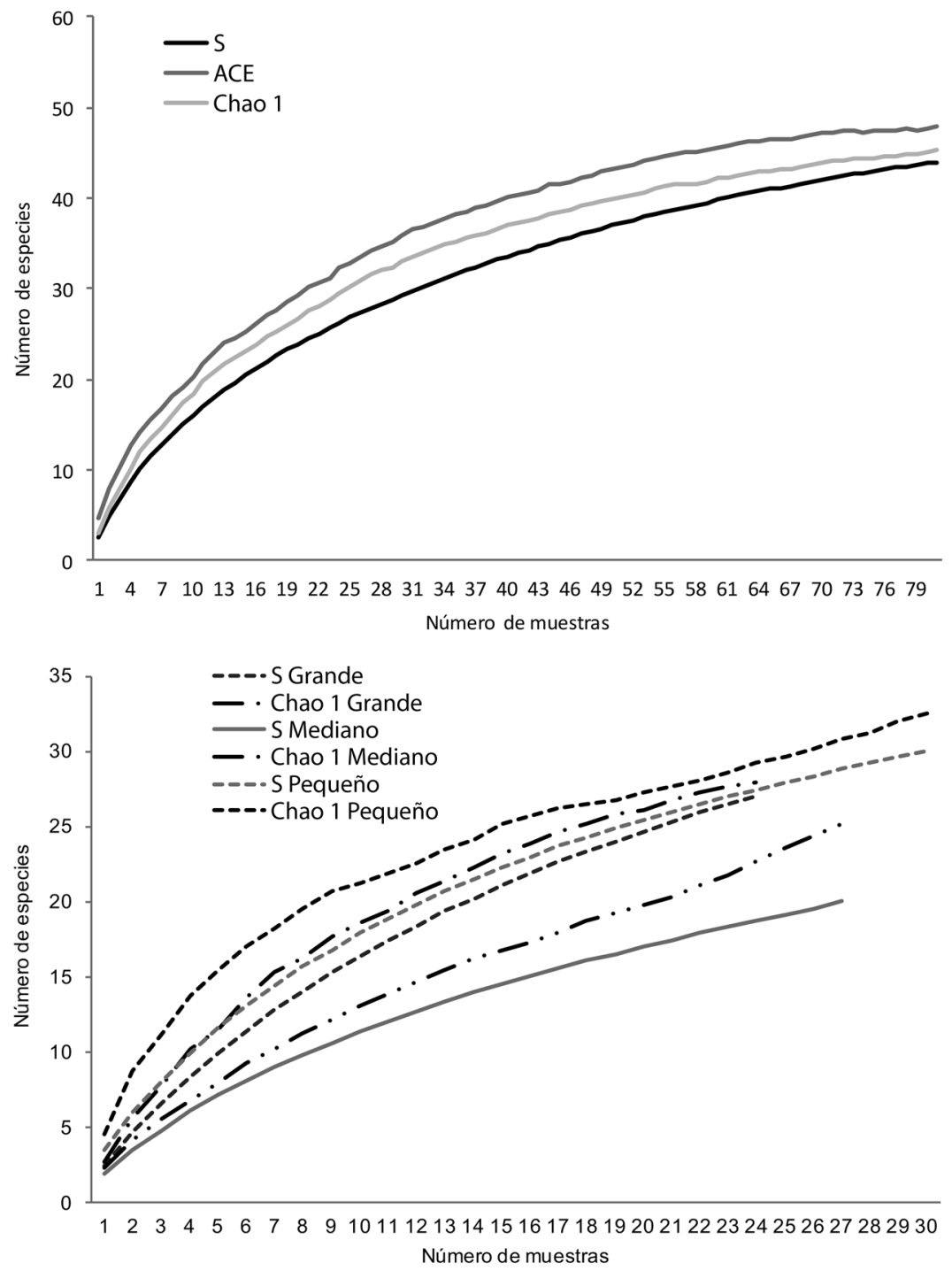

Fig. 2. A. Curva de acumulación de especies de aves en todo el cultivo de palma de aceite; $\mathbf{B}$. a cada clase de altura.

Fig. 2. A. Accumulation rate of bird species related to the entire Oil Palm crop; B. related to each height class.

mientras que en las palmas pequeñas tuvieron mayor dominancia $(\lambda)$ de especies (Cuadro 1). Al comparar entre pares de biotas, la mayor diferencia en la riqueza de especies fue entre palmas grandes y pequeñas $\left(\beta_{\mathrm{W}}\right)$, siendo a la vez más complementarias $\left(\mathrm{C}_{\mathrm{A}, \mathrm{B}}\right)$ (Cuadro 1$)$.

La estructura trófica de las aves asociadas al cultivo de palma de aceite estuvo conformada por ocho grupos y predominaron las especies insectívoras, seguido de las carnívoras, las cuales estuvieron principalmente presentes en palmas pequeñas y medianas, respectivamente $\left(\mathrm{Chi}^{2}=342.4\right.$, valor de $\left.\mathrm{p}<0.001\right)$ (Cuadro 2). Las aves insectívoras más comunes estuvieron representadas por Gymnomystax mexicanus, Crotophaga ani, Tyrannus melancholicus y Cyanocorax violaceus, y las carnívoras por Milvago chimachima, Rupornis magnirostris y Tyto alba. Finalmente, con el análisis de ordenación, se pudo identificar que las asociaciones 


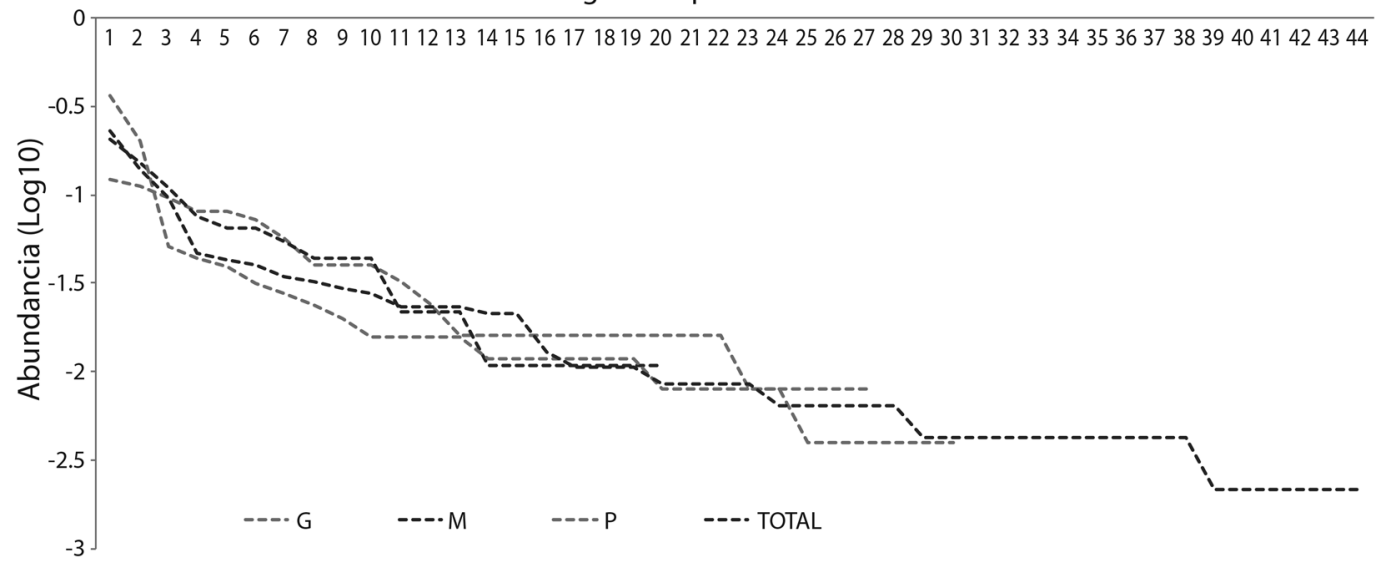

Fig. 3. Modelo de distribución de abundancia de especies de aves asociadas a cultivos de palma de aceite (Elaeis guineensis). Fig. 3. Species abundance distribution model of birds associated to Oil Palm crops (Elaeis guineensis).

CUADRO 2

Grupos tróficos de aves asociadas a cultivos de palma de aceite

TABLE 2

Trophic groups of birds associated of the Oil Palm crop

\begin{tabular}{|c|c|c|c|c|c|c|}
\hline \multirow{2}{*}{ Grupo Trófico } & \multirow{2}{*}{$\mathrm{S}$} & \multicolumn{4}{|c|}{ Abundancia de las especies } & \multirow[b]{2}{*}{$\%$} \\
\hline & & $N(G)$ & $\mathrm{N}(\mathrm{M})$ & $\mathrm{N}(\mathrm{P})$ & N (Total) & \\
\hline CAR & 6 & 28 & 31 & 14 & 73 & 15.60 \\
\hline CAR-CRR & 1 & 2 & 1 & 1 & 4 & 0.85 \\
\hline CRR & 1 & 1 & 2 & 0 & 3 & 0.64 \\
\hline FRU & 8 & 11 & 23 & 14 & 48 & 10.26 \\
\hline GRA & 4 & 4 & 7 & 18 & 29 & 6.20 \\
\hline INS & 21 & 66 & 27 & 200 & 293 & 62.61 \\
\hline NEC & 1 & 0 & 1 & 1 & 2 & 0.43 \\
\hline $\mathrm{OMN}$ & 2 & 12 & 0 & 4 & 16 & 3.42 \\
\hline Total & 44 & 124 & 92 & 252 & 468 & 100.00 \\
\hline
\end{tabular}

CAR: carnívoro, CAR-CRR: carnívoro-carroñero, FRU: frugívoro, FRU-INS: frugívoro-insectívoro, GRA: granívoro, INS: insectívoro, NEC: nectarívoro, OMN: omnívoro.

CAR: carnivore, CAR-CRR: carnivore-scavenger, FRU: frugivore, FRU-INS: frugivore-insectivore, GRA: granivore, INS: insectivore, NEC: nectarivore, OMN: omnivore.

más importantes fueron de aves nectarívoras en palmas pequeñas y medianas; por su parte las aves insectívoras mostraron mayor afinidad a palmas pequeñas y grandes, y las aves carnívoras y carroñeras tuvieron mayor asociación con palmas grandes y medianas (Fig. 4).

\section{DISCUSIÓN}

La abundancia de aves no fue significativamente diferente entre las clases de altura de las palmas. La riqueza total observada (44 especies) refleja que el monocultivo estudiado 

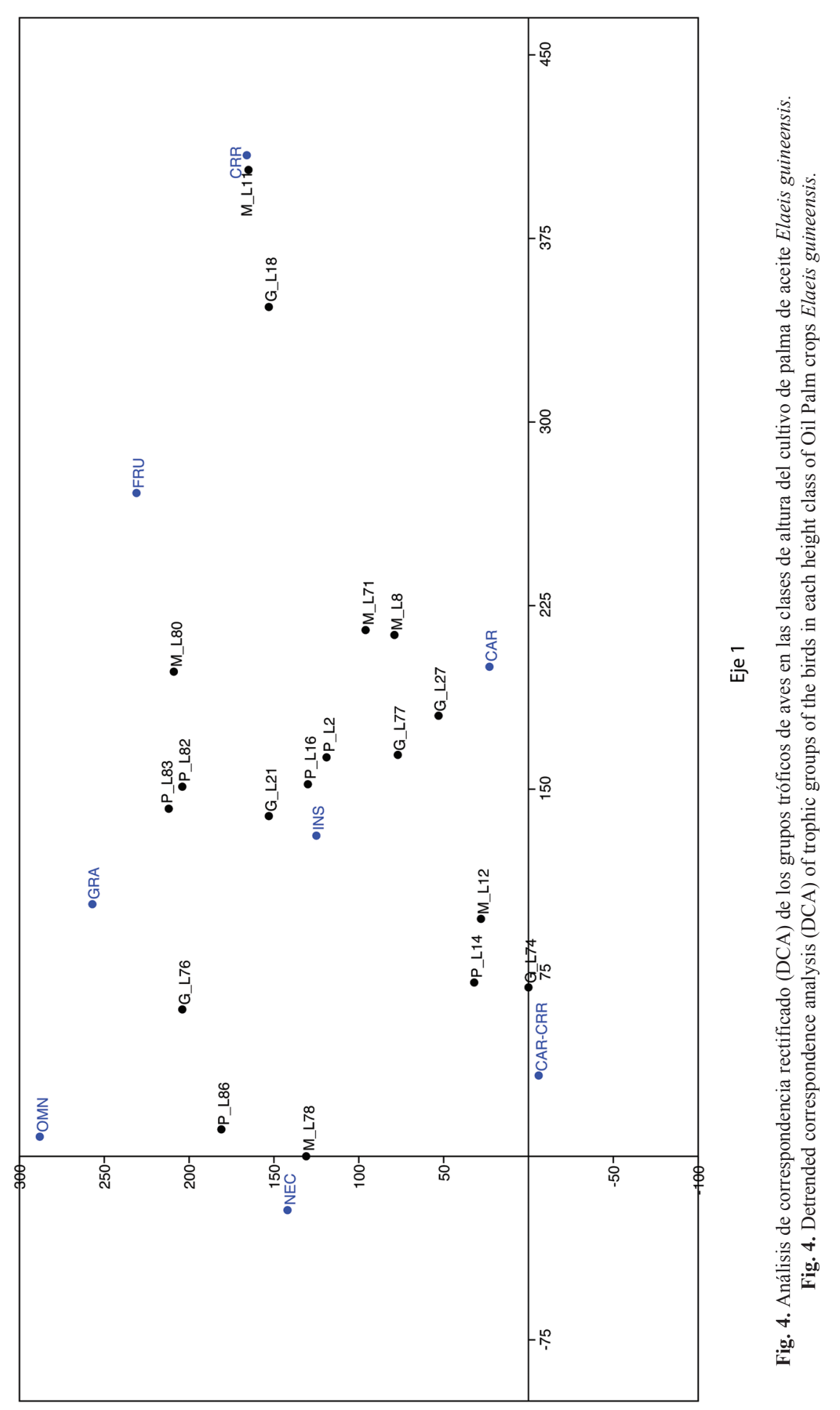

Eje 2 
soportó un bajo número de especies si se tiene en cuenta que representan el $5.78 \%$ de las especies reportadas para la región, el $6.44 \%$ de las especies reportadas para el departamento del Meta, y corresponde al $10.6 \%$ de las especies reportadas para el piedemonte de la Orinoquía colombiana (McNish, 2007; Acevedo-Charry, Pinto-Gómez \& Rangel-Ch, 2014). El estudio como de Enríquez Bernal (2006) también encontró un reducido número de especies de aves asociadas al cultivo de palma de aceite (27 y 29 en palmas pequeñas y grandes), en comparación con otros ecosistemas como bosque de galería y pastizales donde registraron 41 y 26 especies, respectivamente. Nuestros resultados también demostraron que puede presentarse una baja asociación de aves dentro del monocultivo indistintamente de la clase de altura, al considerar que en los recorridos donde no hubo registro de aves $(n=27)$, ocurrieron en igual proporción en sectores de palmas grandes, medianas y pequeñas.

Las curvas de rarefacción estuvieron influenciadas por las especies únicas y dobles, lo que genera la mayoría de las veces que los muestreos se consideren incompletos. Sin embargo, esto no invalida el trabajo, más bien demuestra que son pocos individuos, de las diferentes especies reportadas, que hacen uso de las plantaciones de palmas. La ausencia de un porcentaje considerable de especies de aves propias del piedemonte, puede estar relacionada con la pérdida de bosque, la expansión del monocultivo y los cambios en el uso del suelo en los municipios de Acacias y San Martín de los Llanos, en los cuales se ha reportado un crecimiento en el área cultivada del $10.23 \%$ y $5.67 \%$, respectivamente, entre los años 1988 y 2005 (Pinzón et al., 2009). De igual forma ha ocurrido en Villanueva (Casanare), donde encontraron que el cultivo de palma de aceite ha sido la cobertura de mayor expansión entre el 2002 y el 2012 con respecto a otros usos de suelo, periodo en el cual presentó una ganancia de 1923.15 ha, y que a su vez ha sustituido pastos, bosques de galería y vegetación secundaria, considerados ecosistemas estratégicos para la conservación de la biodiversidad (González \& Romero, 2013).

La siembra a escala industrial ha promovido la remoción de formaciones vegetales originales: bosques altos, palmares de Socratea exorrhiza, vegetación acuática, matorrales y pastizales con restos de bosque (RangelCh \& Minorta-Cely, 2014), a su vez que se han generado eventos de extinción local de las aves asociadas al piedemonte: Anhima cornuta, Neochen jubata, Amazonetta brasilensis, Aburria aburri, Mitu tomentosum, Crax daubentoni, Harpia harpia, Opisthocomus hoazin, Eurypyga helias, Geotrigon linearis, Orthopsittaca manilata, Atene cunicularia, Nyctibius grandis, Eutoxeres aquila, Phaetornis malaris, Pachyramphus cinnamomeus, P. polychopterus, Anthu lutescens, entre otras especies (Olivares, 1982; McNish, 2007; Acevedo-Charry et al., 2014).

El reemplazo de la vegetación original que incluye bosques con un estrato arbóreo de $30 \mathrm{~m}$ (Cassia moschata), por un sistema que a gran escala es uniforme en edad y estructura, de bajo dosel $(13 \mathrm{~m})$, escaso sotobosque y un microclima más severo (Fitzherbert et al., 2008; Luskin \& Potts, 2011), reduce la asociación de especies en comparación con las formaciones vegetales originales (Acevedo-Charry et al., 2014). Romero (2014) al estudiar vegetación arbustiva y arbórea en Barbascal (Meta) donde predominan los cultivos de palma de aceite registró 59 especies de aves de las cuales 49 no fueron registradas en el cultivo de palma. Prescott, Gilory, Haugaasen \& Medina (2016) también sostienen que la riqueza y la diversidad funcional de las aves es menor en palmares de aceite que fueron comparados con remanentes de bosque.

La reducción de la riqueza de aves dentro de este monocultivo es mayor que en otros cultivos, donde se ha reportado mayor número de especies. Por ejemplo, en cultivos de café con sombra se han registrado 141 especies (Bayly \& Gómez, 2010), en arrozales 150 (Cifuentes-Sarmiento \& Castillo, 2016), mientras el presente estudio reporta 44 aves asociadas al cultivo de palma de aceite, y estudios previos 
han registrado 34 y 29 especies en el piedemonte amazónico y Acacias, respectivamente (Enríquez Bernal 2006; Velásquez Valencia, 2009).

La abundancia de individuos fue notablemente mayor en palmas pequeñas. Esta tendencia difiere con otros estudios donde han encontrado mayor abundancia de aves en plantaciones maduras que en palmas jóvenes como en palmares de Indonesia y Filipinas (De Chenon \& Susanto, 2006; Cagod \& Nuñeza, 2012). Se argumenta que las palmas maduras soportan mayor biodiversidad que las palmas recién sembradas porque tienen mayor complejidad estructural, mayor desarrollo de sotobosque y un microclima más conveniente (Azhar et al., 2015; Luskin \& Potts, 2011). En nuestro caso, la remoción de plantas herbáceas y arbustivas del suelo, le resta complejidad al cultivo, la asociación de invertebrados y artrópodos, y con ello recursos potenciales de forrajeo y alimentación para las aves (Cagod \& Nuñeza, 2012).

La estructura trófica de la comunidad estuvo conformada por ocho grupos y las aves insectívoras fueron predominantes. Esta tendencia se ha encontrado en estudios previos y se ha considerado que este conjunto de especies actúan sobre el control de insectos que representan riesgos para el cultivo (Enríquez Bernal, 2006; Achondo et al., 2011; Azhar et al., 2013). Sin embargo, también se ha encontrado que las aves generalistas y frugívoras son abundantes. Azhar et al. (2013) encontraron que las aves frugívoras fueron abundantes en palmas pequeñas, posiblemente porque los lotes donde fueron registradas tuvieron mayor proximidad con árboles nativos, y que la mayoría de los grupos tróficos fueron dominados por pocas especies. Las aves nectarívoras exhibieron mayor sensibilidad al establecimiento de la plantación si se tiene en cuenta la escasa abundancia proporcional de los individuos (Amazilia versicolor). Estudios previos no relacionan la presencia de este grupo trófico con el cultivo de palma, lo que concuerda con la reducción la diversidad funcional de las aves en paisajes dominados por cultivos de palma de aceite (Azhar et al., 2013, Prescot et al., 2016).
Esta investigación destaca que los cultivos de palma de aceite pueden funcionar como un hábitat favorable para un bajo número de especies, en este caso Gymnomystax mexicanus, Crotophaga ani, Milvago chimachima y Rupornis magnirostris, si nos basamos en su abundancia proporcional, pero también pueden funcionar como un hábitat poco amigable o como una barrera de movimiento para el $75 \%$ de las especies que fueron raras dentro de la comunidad, debido a la pérdida y fragmentación del bosque, y porque el aislamiento entre parches de bosque, impide el movimiento de aves, aunque rutinariamente muchas cruzan terrenos inhóspitos (Bélisle, Desrochers \& Fortin, 2001). Este conjunto de especies (el $75 \%$ ) frecuenta principalmente ecosistemas con vegetación arbustiva y arbórea (Hilty \& Brown, 2001), pero la escasa complejidad del cultivo reduce la explotación de recursos y el bajo grado de asociación de las aves dentro de este sistema.

Este estudio encontró que los cultivos de palma de aceite tuvieron una baja asociación de aves, y las especies que alberga, son de amplia distribución y baja importancia de conservación. La variación de altura de las palmas aparentemente no refleja un beneficio para las aves, al observarse que la abundancia de individuos no presentó diferencias significativas entre clases de altura. La expansión acelerada de este monocultivo podría representar un detrimento en la disponibilidad y calidad de hábitat para las aves, si tenemos en cuenta que el $75 \%$ de los miembros de la comunidad fueron raros dentro de la plantación. Con el panorama actual es recomendable que si se decide expandir este monocultivo, se mantenga la vegetación nativa existente, para que estos relictos puedan proporcionar recursos de alimento, refugio, sitios de reproducción y movimiento, a las especies que fueron escasas y raras dentro del cultivo y aquellas que usan este tipo de cobertura.

\section{AGRADECIMIENTOS}

Agradecemos a los integrantes del grupo en Conservación y Manejo de Vida Silvestre 
y al programa de posgrado de la Universidad Nacional de Colombia por su apoyo logístico y académico. A Idea Wild por suministrar equipos y materiales a este proyecto de investigación y al grupo de investigación de Ecología Funcional del INECOL por los aportes al manuscrito, finalmente a los administradores de las fincas La Mejorana y Tierra Viva por el apoyo logístico que nos brindaron durante el trabajo de campo.

\section{RESUMEN}

La expansión del cultivo de palma de aceite ha ocasionado cambios drásticos en los hábitats naturales y modificado la estructura de la vegetación, reduciendo el hábitat disponible para las especies de aves nativas. Se estudiaron las aves asociadas a lotes de palma de aceite de diferentes clases de alturas en el piedemonte de la Orinoquia colombiana, entre agosto 2013 y febrero 2014. Las clases de altura fueron tres: pequeña hasta seis metros, mediana hasta 10 metros y grande hasta 15 metros, en cada lote se censaron las aves mediante transectos lineales y se comparó la composición, riqueza, abundancia y describió la estructura de grupos tróficos. Registramos 468 individuos de 44 especies y 23 familias. Gymnomystax mexicanus, Crotophaga ani y Milvago chimachima fueron las especies más abundantes, las dos primeras representan el $4.5 \%$ de las especies abundantes, el $25 \%$ tuvo abundancias intermedias y el $70.4 \%$ fueron especies raras en el área del cultivo. En términos de la abundancia proporcional de especies de aves, las palmas grandes y medianas fueron más parecidas. La comunidad de aves encontrada en parcelas de palma grande tuvo mayor diversidad y uniformidad (2.63 y 0.8907 , respectivamente), mientras que en las palmas pequeñas hubo mayor dominancia y abundancia de individuos $(0.1825 \mathrm{y}$ 252, respectivamente). En todo el cultivo predominaron aves insectívoras y carnívoras, las cuales tuvieron mayor afinidad por palmas pequeñas y grandes. La palma de aceite puede funcionar como un hábitat favorable para un bajo número de especies de amplia distribución y la altura no parece ser una variable que beneficie de manera significativa la composición, riqueza y abundancia de las especies de aves asociadas a la plantación.

Palabras clave: comunidad de aves, prácticas agrícolas, Orinoquía, complejidad estructural, palma de aceite, grupos tróficos.

\section{REFERENCIAS}

Acevedo-Charry, O., Pinto-Gómez, A., \& Rangel-Ch, J. O. (2014). Las aves de la Orinoquía colombiana: una revisión de sus registros. En J. O. Rangel-Ch (Ed.),
Colombia Diversidad Biótica XIV. La región de la Orinoquía de Colombia (pp. 691-750). Universidad Nacional de Colombia - Instituto de Ciencias Naturales. Bogotá, D.C., Colombia.

Achondo, M. J. M., Casin, L. F., Bello, V. P., Tanalgo, K. C., Agduma, A. R., Bretaña, B. L. P., Mancao, L. S., Salem, J. G. S., \& Supremo, J. P. (2011). Rapid assessment and feeding guilds of birds in selected Ruber and Oil Palm plantations in North Cotabato. Asian Journal of Biodiversity, 2, 103-120.

Andrade Pérez, G. I., Castro Gutiérrez, L. G., Durán Durán, A., Rodríguez Becerra, M., Rudas Lleras, G., Uribe Botero, E., \& Wills Herrera, E. (2009). La mejor Orinoquía que podemos construir Elementos para la sostenibilidad ambiental del desarrollo. Bogotá, Colombia: Universidad de Los Andes y Corporinoquía.

Aratrakorn, S., Thunhikorn, S., \& Donald, P. F. (2006). Changes in bird communities following conversion of lowland forest to oil palm and rubber plantations in southern Thailand. Bird Conservation International, 16, 71-82.

ASD (Agricultural Services and Development). (2006). Aspectos Generales del cultivo de la palma aceitera (Elaeis guineensis Jacq.). San José, Costa Rica: Agricultural Services and Development.

Azhar, B., Lindenmayer, D. B., Wood, J., Fischer, J., Manning, A., McElhinny, C., \& Zakaria, M. (2013). The influence of agricultural system, stand structural complexity and landscape context on foraging birds in oil palm landscapes. Ibis, 155, 297-312.

Azhar, B., Saadun, N., Leong Puana, Ch., Kamarudin, N., Aziz, N., Nurhidayu, S., \& Fischer, J. (2015). Promoting landscape heterogeneity to improve the biodiversity benefits of certified palm oil production: Evidence from Peninsular Malaysia. Global Ecology and Conservation, 3, 553-561.

Bayly, N. J., \& Gómez, C. (2010). Las aves de la Hacienda La Victoria. (Informe Técnico No. CEC01). Bogotá, Col. SELVA: Investigación para la conservación en el Neotrópico, Bogotá.

Bélisle, M., Desrochers, A., \& Fortin, M. J. (2001). Influence of forest cover on the movements of forest birds: a homing experiment. Ecology, 82(7), 1893-1904.

Bibby, C. J., Jones, M., \& Marsden, S. (2000). Expedition field techniques birds survey. London, UK.: Academic Press.

Blydenstein, J. (1967). Tropical savanna vegetation of the Llanos de Colombia. Ecology, 48(1), 1-15.

Bolwing, S., Pomeroy, D., Tushabe, H., \& Mushabe, D. (2006). Crops, trees, and birds: Biodiversity change under agricultural intensification in Unganda's 
farmed landscape. Danish Journal of Geography, 106(2), 115-130.

Buckland, S. T., Anderson, D. R., Burnham, K. P., \& Laake, J. L. (1993). Distance Sampling: Estimating Abundance of Biological Populations. London, UK: Chapman and Hall.

Cagod, B. M., \& Nuñeza, O. M. (2012). Avian species diversity in oil palm plantations of Agusan Del Sur and Compostela Valley, Philippines. AES-Bioflux, $4(2), 85-105$.

Cifuentes-Sarmiento, Y., \& Cantillo Cortés, L. F. (2016). Las alas del arroz: aves acuáticas en cultivos ecoamigables del Valle del Cauca. Cali, Colombia: Asociación Calidris \& Arrocera La Esmeralda S.A.S.

Codesido, M., \& Bilenca, D. (2004). Variación estacional de un ensamble de aves en un bosque subtropical semiárido del Chaco Argentino. Biotropica, 36(4), 544-554.

Correa, H. D, Ruiz, S. L., \& Arévalo, L. M. (2005). Plan de acción en biodiversidad de la cuenca del Orinoco - Colombia / 2005 - 2015 - Propuesta Técnica. Bogotá D.C.

Correa, D. F., \& Stevenson, P. R. (2010). Estructura y diversidad de los bosques de galería en una sabana estacional de los llanos orientales colombianos (Reserva Tomo Grande, Vichada). Orinoquía 14(1), 31-48.

Cousin, J., \& Phillips, R. D. (2008). Habitat complexity explains species-specific occupancy but not species richness in a Western Australian Woodland. Australian Journal Zoology, 56, 95-102.

De Chenon, R., \& Susanto, A. (2006). Ecological observations on diurnal birds in Indonesian oil palm plantations. Journal of Oil Palm Research (Special Issue April), 122-143.

Edwards, D. P., Hodgson, J. A., Hamer, K. C., Mitchell1, S. L., Ahmad, A. H., Cornell, S. J., \& Wilcove, D. S. (2010). Wildlife-friendly oil palm plantations fail to protect biodiversity effectively. Conservation Letter, $3,236-242$.

Enríquez Bernal, L. M. (2006). Estructura y composición de la comunidad de aves asociadas a plantaciones de palma de aceite (Elaeis guineensis Jacq.), en Agropecuaria La Loma (Acacias - Meta) (Tesis de Pregrado). Pontificia Universidad Javeriana, Bogotá, Colombia.

Fedepalma (Federación Nacional de Cultivadores de Aceite de Palma). (2007). Localización geográfica de cultivos y planta de beneficio. Sistema de Información Estadística del Sector Palmero. Recuperado de www. fedepalma.org

Fitzherbert, E. B., Struebig, M. J., Morel, A., Danielsen, F. Brühl, C. A., Donald, P. F., \& Phalan, B. (2008). How will oil palm expansion affect biodiversity?. Trends in Ecology and Evolution, 23(10), 538-545.

García-Ulloa, J., Sloan, S., Pacheco, P., Ghazoul, J., \& Pin Koh, L. (2012). Lowering environmental cost of oilpalm expansion in Colombia. Conservation Letters, $5,366-375$

González, L. M., \& Romero, A. P. (2013). Análisis multitemporal de los cambios de cobertura de la tierra $e$ incidencia del cultivo de palma de aceite en el territorio del municipio de Villanueva Casanare (Tesis de Maestría). Pontificia Universidad Javeriana, Bogotá, Colombia.

Hilden, O. (1965). Habitat selection in birds: A review. Annales Zoologici Fennici, 2(1), 53-75.

Hilty, S., \& Brown, W. L. (2001). Guía de las aves de Colombia. Colombia: American Bird Conservancy-ABC.

Koh, L. P., \& Ghazoul, J. (2008). Biofuels, biodiversity, and people: understanding the conflicts and finding opportunities. Biological Conservation, 141(10), 2450-2460

Krebs, Ch. J. (1999). Ecological Methodology. CA. E.U.: Addison Wesley Longman.

Luskin, M. S., \& Potts, M. D. (2011). Microclimate and habitat heterogeneity through the oil palm lifecycle. Basic and Applied Ecology, 12, 540-551.

MacArthur, R. H., \& MacArthur, J. W. (1962). On bird species diversity. The American Naturalist, 96(888), 167-174.

Magurran, A. (1988). Ecological diversity and its measurement. New Jersey, USA: Princeton University Press.

McNish, T. (2007). Las aves de los Llanos de la Orinoquía. Bogotá, Colombia: M\&B Ltda.

Miller, J. R., \& Cale, P. (2000). Behavioral mechanism and habitat use by birds in a fragmented agricultural landscape. Ecological Applications, 10(6), 1732-1748.

Minorta-Cely, V., \& Rangel-Ch., J. O. (2014). Clima de la Orinoquía colombiana. En J. O. Rangel-Ch (Ed.), Colombia Diversidad Biótica XIV. La región de la Orinoquía de Colombia (pp. 153-206). Bogotá, D.C., Colombia: Universidad Nacional de Colombia - Instituto de Ciencias Naturales.

Mora Fernández, C., Castellanos Castro, C., Cardona Cardozo, A., Pinzón Pérez, L., \& Vargas, O. (2011). Historia de transformación del paisaje, de la cuenca baja del río Pauto, Casanare (Colombia). En T. L. Sicard (Ed.), Mamiferos, Reptiles y Ecosistemas del Bloque Cubiro (Casanare). Educación Ambiental para la Conservación. Bogotá. Colombia: Instituto de Estudios Ambientales, Universidad Nacional de Colombia. 
Moreno, C. (2001). Métodos para medir la biodiversidad. M\&T-Manuales y Tesis SEA. Vol. 1. Zaragoza, España.

Nájera, A., \& Simonetti, J. A. (2010). Enhancing Avifauna in Commercial Plantations. Conservation Biology, 24(1), 319-324.

Olivares, A. (1982). Aves de la Orinoquía colombiana (2da Edición). Bogotá, Colombia: Instituto de Ciencias Naturales Universidad Nacional de Colombia.

Pinzón, F., Pardi, M. S., Trejos Serrato, J., \& Midori Saito, S. (2009, Abril). Uso de técnicas de percepción remota para la estimación del incremento de áreas cultivadas de palma aceitosa y su relación con el conflicto armado en el Departamento del MetaColombia. Anais En XIV Simpósio Brasileiro de Sensoramiento Remoto. Natal, Brasil.

Prescott, G. W., Gilory, J. J., Haugaasen, T., \& Medina Uribe, C. A. (2016). Reducing the impacts of Neotropical oil palm development on functional diversity. Biological Conservation, 197, 139-145.

Rangel-Ch, O., \& Minorta-Cely, V. (2014). Tipos de vegetación de la Orinoquía colombiana. En J. O. Rangel-Ch (Ed.), Colombia Diversidad Biótica XIV. La región de la Orinoquía de Colombia. Bogotá, Colombia: Universidad Nacional de Colombia - Instituto de Ciencias Naturales.

Restall, R., Rodner, C., \& Lentino, R. (2006). Birds of northern South America. London: Christopher Helm.
Rippstein, G., Escobar, G., \& Motta, F. (2001). Agroecología y biodiversidad de los Llanos Orientales de Colombia. Cali, Colombia: Centro Internacional de Agricultura Tropical (CIAT) \# 322.

Romero, N. (2014). Efecto de la estructura de la cobertura natural sobre la composición de aves de sotobosque en áreas dominadas por cultivos de palma africana en San Martin, Meta (Tesis de pregrado). Universidad Nacional de Colombia, Bogotá, Colombia.

Roundtable on Sustainable Palm Oil (RSPO) (2014). Principios y Criterios para la producción de aceite de palma sostenible. Documento Borrador de la Interpretación Nacional (IN) para Colombia del estándar RSPO 2013 para consulta pública. Fedepalma.

Umaña-Villaveces, A. M., Murillo, J. I., Restrepo-Calle, S. \& Álvarez-Rebolledo, M. (2009). Indicadores de seguimiento de la biodiversidad en el piedemonte orinoquense, sabanas y bosques asociados al norte del río Guaviare: Aves. En M. H. Romero (Eds.), Informe sobre el estado de la biodiversidad en Colombia 2007-2008: Piedemonte orinoquense, sabanas y bosques asociados al norte del río Guaviare (pp. 48-78). Bogotá, D.C.: Instituto de Investigación de Recursos Biológicos Alexander von Humboldt.

Velásquez Valencia, A. (2009). Estructura de la comunidad de aves en sistemas de producción del piedemonte amazónico (Tesis de Maestría). Universidad Nacional de Colombia, Colombia. 